\title{
Reliable First-Principles Alloy Thermodynamics via Truncated Cluster Expansions
}

\author{
Nikolai A. Zarkevich* and D.D. Johnson \\ Departments of Physics and Materials Science $\&$ Engineering, \\ and Frederich Seitz Materials Research Laboratory, \\ University of Illinois, Urbana-Champaign, IL 61801
}

(Dated: April 4, 2003)

\begin{abstract}
In alloys cluster expansions (CE) are increasingly used to combine first-principles electronicstructure calculations and Monte Carlo methods to predict thermodynamic properties. As a basis-set expansion in terms of lattice geometrical clusters and effective cluster interactions, the CE is exact if infinite, but is tractable only if truncated. Yet until now a truncation procedure was not well-defined and did not guarantee a reliable truncated $\mathrm{CE}$. We present an optimal truncation procedure for $\mathrm{CE}$ basis sets that provides reliable thermodynamics. We then exemplify its importance in $\mathrm{Ni}_{3} \mathrm{~V}$, where the $\mathrm{CE}$ has failed unpredictably, and now show agreement to a range of measured values, predict new low-energy structures, and explain the cause of previous failures.
\end{abstract}

PACS numbers: 05.10.-a,64.70.Kb,81.30.Bx

The cluster expansion (CE) is increasingly used as a valuable tool for predicting and interpreting thermodynamic effects in a wide class of materials and problems, including precipitation [1, 2, 3, 4], solubility limits 5], ionic diffusion [6], surface alloying [7] and patterning [8], vacancy [9] and chemical [3, 10] ordering. As a means for multiscaling based on density-functional theory (DFT) electronic-structure energetics, the $\mathrm{CE}$ is a basis-set expansion in $n$-body clusters (associated with $n$ Bravais lattice points) and effective cluster interactions (ECI) that specify configurational energies. Except for implicit DFT errors in the energy database, the CE is exact for an infinite basis, but impractical if not vastly truncated [1, 12]. Although there are many successes, a truncated CE can and has unpredictably failed.

We present a new method for an optimal truncation of the basis set that gives reliable thermodynamics. We then detail its importance in face-centered-cubic (fcc) $\mathrm{Ni}_{3} \mathrm{~V}$, a system with order-disorder transition from disordered $A 1$ phase to ordered $\mathrm{DO}_{22}$ phase at $T_{c}$ of $1318 \mathrm{~K}$ 13]. Previous $\mathrm{CE}$ for $\mathrm{Ni}_{3} \mathrm{~V}$ [14, 15, 16] had errors of $40-1000 \%$ for a range of thermodynamic properties, prompting a search for missing physics [16]. We show that our new method allows more reliable predictions, including that of key low-energy configurational excitations. As a synopsis, we compare in Table \ our CE results, along with the previous ones, with experimental values of $T_{c}$ and $\Delta E_{S R O}^{L 1_{2}-D O_{22}}$, the energy difference between $\mathrm{DO}_{22}$ and metastable $\mathrm{L}_{2}$ as assessed from the short-range order (SRO) measurements [17, 18]. The new CE now agrees with a range of experimentally assessed values (more below). We find that prior failure in $\mathrm{Ni}_{3} \mathrm{~V}$ is due to inappropriate truncation of the cluster basis set and overfitting to get the ECI - underscoring again the need for careful application of basis-set methods. We have tested this new CE method on a few cubic and noncubic binaries and ternaries and found it to be especially important when multibody ECI are significant.
Cluster Expansions: Any alloy configuration may be represented by a set of occupation variables $\left\{\xi_{p}^{\alpha}\right\}$, with $\xi_{p}^{\alpha}=1(0)$ if the site $p$ is (is not) occupied by an $\alpha$-atom. Composition $c^{\alpha}$ is the thermal- and site-average of $\left\{\xi_{p}^{\alpha}\right\}$ with $0 \leq c^{\alpha} \leq 1$. The energy of any atomic configuration $\sigma$ can be written in a CE [1] using the $n$-body ECI $V_{f}^{(n)}$ :

$$
E_{C E}(\sigma)=V^{(0)}+\sum_{n, f, d} V_{f}^{(n)} \bar{\Phi}_{f d}^{(n)}(\sigma)
$$

Sums are over symmetry-distinct $(n, f)$ and equivalent $\left(d=1, \ldots, D_{f}^{(n)}\right.$, the degeneracy) clusters. A CE basis can be also presented as a product of orthonormal Chebychev polynomials based on $\xi_{p}^{\alpha}$ [1]. The $n$-site correlation function $\bar{\Phi}_{f d}^{(n)}=\left\langle\xi_{p^{\prime}} \xi_{p^{\prime \prime}} \ldots \xi_{p^{(n)}}\right\rangle$ is given by an ensemble average over the fixed sets $\{p\}_{f d}^{(n)}$ defining the $n$-body clusters of type $(f, d)$, see Fig. 1] When evaluated above $T_{c}, \bar{\Phi}^{(2)}$, for example, are related to the SRO. If the ECI are known, then the energy of any configuration can be predicted.

A $\mathrm{CE}$ can be truncated if there is rapid convergence of the ECI $V_{f}^{(n)}$ with increasing distance $r$ (e.g., as measured by cluster radius of gyration or circumscribed sphere) and with increasing number of sites $n$ in a cluster $f$, i.e. smaller $n$-body clusters are more physically important. Also $V_{f}^{(n)}$ for $n>n_{0}$ uncorrelated sites have their contributions to (11) suppressed by $\bar{\Phi}^{(n)} \sim c^{n}$, i.e. $V^{(n)} \Phi^{(n)} \rightarrow 0$, and can be neglected. The magnitudes

TABLE I: New truncated $\mathrm{CE}\left(\mathrm{CE}_{2}\right.$ and $\left.\mathrm{CE}_{3}\right)$ and experimental [17] values of $T_{c}$ (Kelvin) and the $\Delta E_{S R O}^{L 1_{2}-D O_{22}}$ (meV/atom) assessed from SRO, along with the former $\mathrm{CE}$ [14] and CPA [19] results. Details in text.

\begin{tabular}{l|cc|l|c|c}
\hline & $\mathrm{CE}_{2}$ & $\mathrm{CE}_{3}$ & Expt. & old CE & $\mathrm{CPA}$ \\
\hline$T_{c}(\mathrm{~K})$ & 1335 & 1370 & 1318 & 1900 & \\
$\Delta E_{S R O}^{L 1_{2}-D O_{22}}$ & $22 \pm 16$ & $17 \pm 15$ & $12 \pm 5$ & 101 & $7-12$ \\
\hline
\end{tabular}


of $V^{(n)}$ typically become smaller for larger $n$, although for some systems ECI convergence is not rapid: such is $\mathrm{Li}_{x} \mathrm{NiO}_{2}$ where Jahn-Teller distortions control Li-vacancy ordering and ionic conduction and are reflected only in long-range multibody ECI 20]. For a truncated CE, ECI are obtained via structural inversion [21, 22] at fixed $c$ for $c$-dependent (canonical) ECI or versus $c$ for $c$-independent (grand-canonical) ECI; these sets of ECI are related 23, 24]. First, a set of $N$ fully-ordered (few atoms per cell) structures is somehow chosen and their DFT energies $E_{D F T}^{i}(i=1 . . N)$ are calculated. Then, a set of $M$ clusters $(M<N)$ is somehow picked for use in (11) and $\bar{\Phi}$ are calculated for each structure. A system of $N$ linear equations (1) with $M$ unknown ECI is solved by least-squares (LS) fitting - which unavoidably includes DFT errors in energy differences. As is obvious, the sets of structures and clusters used to get the ECI are not uniquely defined.

New Method: Here we propose a method that, given a set of structural energies, unambiguously defines a set of clusters (and ECI) to provide an optimal truncated CE and yield reliable thermodynamics. First, we note that if $V^{(n)}\left(r>r_{0}^{(n)}\right) \equiv 0$, the truncated CE basis with local compact support that includes all clusters in $r_{0}^{(n)}$ is $l o$ cally complete and exact; whereas, if $V^{(n)}\left(r>r_{0}^{(n)}\right) \approx 0$, this truncated CE is approximate and has an error. With no a priori knowledge of which clusters are required to represent well a given alloy, the CE error is minimal, in a Rayleigh-Ritz variational sense, if all admissible $n$ body clusters (basis functions) of smaller spatial extent $\left(r \leq r_{0}^{(n)}\right)$ are included before the larger ones. In brief, having $N E_{D F T}^{i}$ to be fitted, we can establish a variational CE basis by simple rules ad vitam aut culpam that implement easily computationally:

1. If an $n$-body cluster is included, then include all $n$-body clusters of smaller spatial extent.

2. If a cluster is included, include all its subclusters.

3. To prevent both under- and over-fitting, minimize [25, 26] the cross-validation (CV) error 27, 28]:

$$
C V^{2}=\frac{1}{N} \sum_{i=1}^{N}\left(E_{D F T}^{i}-E_{C E^{\prime}}^{(i)}\right)^{2} .
$$

$E_{C E^{\prime}}^{(i)}$ in (2) is predicted by a fit to $N-1$ DFT energies excluding $E_{D F T}^{i}$, rather than to all $N$ as in a LS fit. (This is an "exclude 1" CV, whereas an "exclude 0" CV is a LS fit.) While LS measures the error in reproducing known values of $E_{D F T}^{i}$, CV error estimates an uncertainty of predicted values. Both too few (underfitting) or too many (overfitting) parameters give poor prediction. The new Rule 1, with well-established Rule 2, now makes it easy to define uniquely all clusters in a truncated, variational CE basis by the number of $n$-body clusters (or the size of the largest $n$-body cluster) for each $n \leq n_{0}$. In

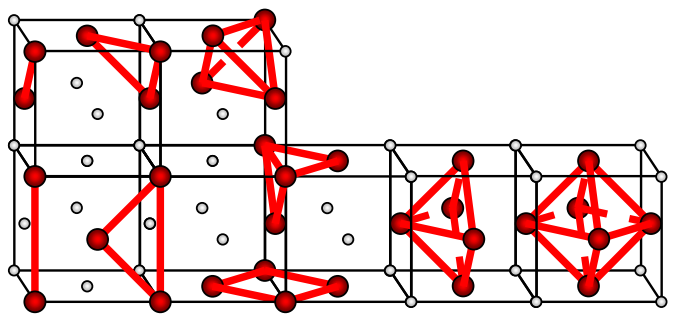

FIG. 1: The 2- to 6-body clusters for $1^{\text {st }}$ and $2^{\text {nd }}$ fcc neighbors. Including clusters up to 4-body in upper set (6-body in both sets) form a locally complete basis in the range of $1^{s t}\left(2^{\text {nd }}\right)$ neighbors. These clusters form tetrahedron (tetrahedron-octahedron) approximations used in clustervariation method [11].

particular, Rules 1 and 2 permit a hierarchy of ranges for $n$-body clusters, i.e., $r_{0}^{(n)} \geq r_{0}^{(n+1)}$ for all $n$, giving a locally complete basis for strict equalities, while the inequality (e.g., more 2-bodies, fewer 3-bodies, even fewer 4-bodies, etc.) allows for fewer clusters. Because shorterranged and lower-order ECI are more important, Rule 1 [Rule 2] prohibits excluding more important ECI and transferring their weight to less important longer-ranged [higher-order] ones.

Once constructed, an optimal CE can be used to predict energy of any structure within the CV error. The $\mathrm{CE}$ is valid if the lowest structural energies (including the ground-state) and fully-disordered state energies are correct within the accuracy given by the CV error. A valid optimal $\mathrm{CE}$ provides reliable thermodynamics.

Application: We now construct and assess the new canonical $\mathrm{CE}$ for $\mathrm{Ni}_{3} \mathrm{~V}$ based on 45 fully-relaxed structural DFT energies, with relative accuracies of $\sim 1 \mathrm{meV} /$ atom [35]. A selected set of energies is given in Table III

To examine effects of the new truncation method on prediction, we first limit the $\mathrm{CE}$ basis to pairs only and find that the pairs-only $\mathrm{CV}$ is minimal for 2 (nearest and next-nearest) pair interactions, see Fig. 2 Within this range, the symmetry-distinct clusters are two pairs,

TABLE II: Selected $\mathrm{Ni}_{3} \mathrm{~V}$ energies (meV/atom) from DFT and $\mathrm{CE}$ relative to $E_{D F T}^{D 0_{22}}$, along with those of disordered state, its approximation by $S Q S_{16}$ [29], and (001) APB.

\begin{tabular}{l|r|rr|rr}
\hline Structure & $E_{D F T}$ & $E_{C E_{2}}$ & $\delta E_{C E_{2}}$ & $E_{C E_{3}}$ & $\delta E_{C E_{3}}$ \\
\hline$D 0_{22}\left(1 \frac{1}{2} 0\right)$ & 0.0 & 7.1 & 7.1 & 10.5 & 10.5 \\
$D 0_{23}\left(1 \frac{1}{4} 0\right)$ & 25.4 & 31.6 & 6.2 & 35.8 & 10.4 \\
& 33.7 & 31.6 & -2.1 & 35.8 & 2.1 \\
$L 1_{2}(100)$ & 101.2 & 56.1 & -45.1 & 61.1 & -40.1 \\
& 147.8 & 170.6 & 22.8 & 154.7 & 6.9 \\
$\mathrm{SQS}_{16}$ & 155.4 & 135.8 & -19.7 & 152.0 & -3.5 \\
\hline Disordered & $\mathrm{n} / \mathrm{a}$ & 109.8 & & 115.1 & \\
$(001) A P B$ & 101.6 & 98.0 & -3.6 & 101.1 & -0.5 \\
$L 1_{2}-D 0_{22}$ & 101.2 & 49.0 & -52.2 & 50.5 & -50.7 \\
$D 0_{23}-D 0_{22}$ & 25.4 & 24.5 & -0.9 & 25.3 & -0.1 \\
\hline
\end{tabular}




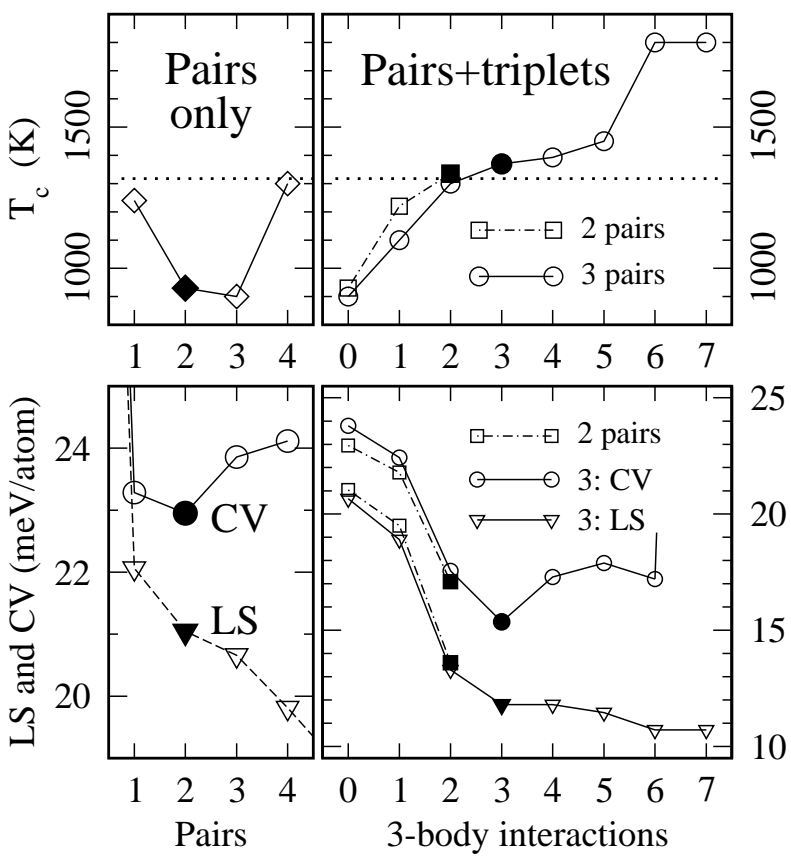

FIG. 2: (upper) The predicted and observed (dotted line) $T_{c}(\mathrm{~K})$, and (lower) the $\mathrm{CV}$ and LS errors (meV/atom) vs. ECI truncation. Results are for CE with pairs-only (left) and for 2 and 3 pairs with a number of 3-body ECI (right). The minimal CV and LS are given by filled symbols.

two 3-bodies, three 4-bodies, a 5-body pyramid and a 6-body octahedron, see Fig. [1 The CE with two pair and two triplet interactions (denoted $\mathrm{CE}_{2}$ ) with minimal $\mathrm{CV}$ of $15.5 \mathrm{meV}$ within this range gives $T_{c}$ at $1335 \mathrm{~K}$, near the observed $1318 \mathrm{~K}$. Including tetrahedron does not significantly alter $T_{c}$, as expected for a 4-body cluster at $c=1 / 4$, as $c^{4} \ll 1$ and $V^{(4)} \Phi^{(4)} \rightarrow 0$. For optimal truncation, including the most compact 4-body cluster is necessary before including any other more extended 4 -body or higher-order clusters. Within our set of 45 arbitrary structures only one $(147.8 \mathrm{meV} /$ atom in Table III) has contribution from the most compact 4-body cluster, so any $\mathrm{CE}$ including this cluster has formally infinite (thus not minimal) CV, so an optimal CE should contain pairs and triplets only. Indeed, the optimal CE (denoted $\mathrm{CE}_{3}$ ), see Fig. 2 includes 3 pair and 3 triplet interactions and yields $T_{c}$ of $1370 \mathrm{~K}$, again near the observed value and well within the CV error of $\pm 15.2 \mathrm{meV}$. Both $\mathrm{CE}_{2}$ and $\mathrm{CE}_{3}$ are examples of the localized $\mathrm{CE}$ hierarchy allowed by Rules 1-3. Notably, we find no failure or ill-description of thermodynamics for optimal truncation embodied in the new rules, see Table $\square$

Moreover, we predicted from these optimal CE that $\mathrm{Ni}_{3} \mathrm{~V}$ has numerous metastable long-period superstructures (LPS) of the type $\left\langle 0 \frac{1}{2 m} 1\right\rangle$ with $m \geq 1$. The $D 0_{22}$ ground state is $m=1, D 0_{23}$ is $m=2$, and $L 1_{2}$ is $m=\infty$, see Table II We then confirmed by direct DFT calculations that over 23 metastable structures are between
$\mathrm{DO}_{22}$ and $L 1_{2}$. Clearly, structural energy differences then will be sensitive to thermal antisites or partial-order, i.e. chemical environments that distinguish $\mathrm{DO}_{22}$ from other low-energy structures.

The relative energies of $\mathrm{DO}_{22}$ and $\mathrm{DO}_{23}$, which can be viewed as $D 0_{22}$ with (001) APBs, give an estimate of the (001) APB energy per site of the antiphase plane: $E^{A P B}=4\left[E^{D 0_{23}}-E^{D 0_{22}}\right]$. In Table III our calculated $E_{D F T}^{A P B}=101.6 \mathrm{meV}$ and $\mathrm{CE}_{3}$-predicted $E_{C E}^{A P B}=$ $101.1 \mathrm{meV}$ agree at perfect long-range order. However, binaries with first-order transitions have order parameters $\eta$ (defined in 19]) that jump from 0 to $0.7-0.9$ at $T_{c}$. For partial order below $T_{c}$ as in experiment, we predict that $E_{C E}^{A P B}(\eta)$ are 81,65 , and $50 \mathrm{meV}$ for $\eta$ 's of $0.9,0.8$, and 0.7 , respectively. From superdislocation separation measurements, assessed values are $52 \pm 20 \mathrm{meV}$ at $273 \mathrm{~K}$ and $55 \pm 18 \mathrm{meV}$ at $900 \mathrm{~K} \mathrm{[17]}$, with roughly constant $\eta<1$ due to lack of kinetics.

The real-space Warren-Cowley SRO parameters $\alpha_{l m n}$ were calculated using our $\mathrm{CE}$ within Monte Carlo at $T=1.04 T_{c}$, as in experiment. The proper way to compare calculated SRO to experimental data is in [4]. Full details of the agreement between calculated and experimental $\alpha_{l m n}$ will be given elsewhere. However the energetics associated with SRO given by $\Delta E_{S R O}^{L 1_{2}-D O_{22}} \approx$ $k_{B} T\left(\alpha^{-1}(100)-\alpha^{-1}\left(1 \frac{1}{2} 0\right)\right) / 16 c(1-c)$ can be directly estimated from the calculated $\alpha(\mathbf{k})$ at $\left\{1 \frac{1}{2} 0\right\}$ and $\{100\}$ k-points, as done experimentally [17]. We obtain $17 \pm$ $15 \mathrm{meV}$ for $\mathrm{CE}_{3}$ at $1392 \mathrm{~K}$, now in agreement with experiment 17] and coherent-potential approximation (CPA) results [19], see Table【 Our results confirm the CPA explanation for $\mathrm{Ni}_{3} \mathrm{~V}$ SRO energetics and the discrepancy between $T=0 \mathrm{~K} \mathrm{DFT}$ results and measurements as arising from the strong dependence of $E^{L 1_{2}}(\eta)$ on the state of partial order [19].

Finally, we discuss issues that led to previous poor $\mathrm{Ni}_{3} \mathrm{~V}$ results. For the range of ECIs included in our truncated $\mathrm{CE}$ basis sets, $\Delta E_{C E}^{L 1_{2}-D 0_{22}} \equiv\left[E_{C E}^{L 1_{2}}-E_{C E}^{D 0_{22}}\right]=$ $2\left[E_{C E}^{D 0_{23}}-E_{C E}^{D 0_{22}}\right] \equiv E^{A P B} / 2$, as verified in Table $\llbracket$ So for a truncated CE, $L 1_{2}$ can be viewed also as a (001) APB in $D 0_{22}$. Other LPS, e.g., with $E_{D F T}=33.7 \mathrm{meV} /$ atom and $E_{D F T}^{D O_{23}}=25.4 \mathrm{meV} /$ atom in Table $\amalg$ also have indistinguishable energies within the truncated CE. This observation has great import in $\mathrm{Ni}_{3} \mathrm{~V}$. Table III shows that $\Delta E_{C E}^{L 1_{2}-D O_{22}}$ and $\Delta E_{D F T}^{L 1_{2}-D O_{22}}$ are not equal! This implies again that there is a strong configurational dependence of partially-ordered $L 1_{2}$ energy on $\eta$, as indeed shown by CPA calculations [19]. Because $L 1_{2}$ is highly metastable with respect to $D 0_{22}$, a truncated $\mathrm{CE}$ will be suspect versus $\eta$ (particularly for $\eta \sim 1$ ) unless all clusters that distinguish $L 1_{2}$ from $D 0_{22}$, and similar LPS, are included in the basis. In Refs. 14, 15, 16, $\Delta E_{C E}^{L 1_{2}-D O_{22}}$ at $0 \mathrm{~K}$ was forced to coincide with $\Delta E_{D F T}^{L 1_{2}-D O_{22}}=101 \mathrm{meV}$ by overfitting and including certain 3-and 4-body clusters arbitrarily that created an invalid $\mathrm{CE}$ and hence 
inaccurate energetics. Our truncated CE properly describes the observed thermodynamics, but not highenergy and ill-described structures like fully-ordered $L 1_{2}$ that are unimportant for thermodynamics, as evidenced in Table II Of course, calculating more DFT structural energies and properly extending the CE basis to include critical $n$-body clusters would describe everything more reliably.

Generally speaking, neglecting stronger interactions and assigning their weight to weaker and less physically important longer-range [or higher-order] ones, i.e. violating Rule 1 [or Rule 2], leads to inaccurate predictions of energetics. Overfitting (neglecting Rule 3) results in large errors in predicted energies, which were not used in the fit. Combined violations can result in dramatic failures: for example, previous CE for Ni-V 14] overfitted energies (violating Rule 3) and included, e.g., a longerranged 4-body before the most compact one (violating Rule 1) - hence the disagreement with experiment and CPA. Previous CE results are generally valid if only Rules 1 and 2 were obeyed with no large overfitting; in such cases, minimizing the $\mathrm{CV}$ error leads only to a moderate improvement of accuracy. The optimal CE basis truncation presented has an error that is variational, which is not necessarily the case for other basis-set truncation and reduction methods.

In summary, the cluster-expansion method is a valuable first-principles tool for predicting and interpreting thermodynamic behavior in alloys. With convergence and reliability in mind, we presented a simple variational method for the optimal truncation of the clusterexpansion basis set. We presented the method as a set of rules that are computationally easy to implement. For a given set of DFT structural energies and no a priori knowledge of which clusters are needed to represent a particular alloy well, our truncation method provides a unique optimal choice of clusters based on their contribution to thermodynamics and variational reduction in error, avoids choosing clusters by intuition, and gives reliable thermodynamic predictions. We exemplified the importance of this new approach in fcc $\mathrm{Ni}_{3} \mathrm{~V}$ by predicting important new metastable structures and by showing agreement with order-disorder temperature, anti-phase boundary energy, and short-range order energetics, all quantities missed by previous cluster-expansion applications. We also elucidated the origin of the previous failures. Without a priori information, the new clusterexpansion strategy allows reliable thermodynamic predictions in alloys.

We acknowledge support from the NSF through a FRG at the Materials Computation Center (DMR-99-76550) and an ITR (DMR-0121695), the U.S. Dept. of Energy through the Frederick Seitz Materials Research Laboratory (DEFG02-91ER45439) and an IBM SURS grant.
* Electronic address: zarkevic@uiuc.edu

[1] V. Vaithyanathan, C. Wolverton, and L.Q. Chen, Phys. Rev. Lett. 88, 125503 (2002).

[2] C. Wolverton and V. Ozoliņš, Phys. Rev. Lett. 86, 5518 (2001).

[3] N.A. Zarkevich and D.D. Johnson, Phys. Rev. B 67, 064104 (2003).

[4] N.A. Zarkevich, et al., Acta Mater. 50, 2443 (2002).

[5] V. Ozoliņš and M. Asta, Phys. Rev. Lett. 86, 448 (2001).

[6] A. Van der Ven, et al., Phys. Rev. B 64, 184307 (2001).

[7] R. Drautz, et al., Phys. Rev. Lett. 87, 236102 (2001).

[8] B.D. Krack, et al., Phys. Rev. Lett. 88, 186101 (2002).

[9] G. L. Hart and A. Zunger, Phys. Rev. Lett. 87, 275508 (2001).

[10] S. Müller and A. Zunger, Phys. Rev. Lett. 87, 165502 (2001).

[11] J.M. Sanchez, et al., Physica 128 A, 344 (1984).

[12] J.M. Sanchez, Phys. Rev. B 48, 14013 (1993).

[13] H. Okamoto, ed., Desk Handbook: Phase Diagrams for Binary Alloys (ASM Int., Materials Park, Ohio, 2000).

[14] C. Wolverton, et al., Phys. Rev. B 49, 16058 (1994).

[15] Z. Lu and A. Zunger, Phys. Rev. B 50, 6626 (1994).

[16] C. Wolverton and A. Zunger, Phys. Rev. B 52, 8813 (1995).

[17] A. Finel, et al., in Metallic Alloys: Experimental and Theoretical Perspectives, edited by J. Faulkner and R. Jordan (Kluwer, Dordrecht, 1994), vol. 256 of NATO Advanced Study Institute, Series B: Physics, pp. 215-224.

[18] M. Barrachin et al., Phys. Rev. B 50, 12980 (1994).

[19] D.D. Johnson et al., Phys. Rev. B 62, R11917 (2000).

[20] M.E. Arroyo y de Dompablo, et al., Phys. Rev. B 63, 144107 (2001).

[21] D. de Fontaine, Solid State Phys. 47, 33 (1994).

[22] D.D. Johnson, in Encyclopedia of Materials: Science 8 Technology, edited by K.H.J. Buschow et al. (Elsevier, NY, 2001).

[23] M. Asta, et al., Phys. Rev. B 44, 4907 (1991).

[24] C. Wolverton, et al., Phys. Rev. B 44, 4914 (1991).

[25] M.H.F. Sluiter, et al., Phys. Rev. B 53, 6137 (1996).

[26] A. van de Walle and G. Ceder, J. of Phase Equilibria 23, 348 (2002).

[27] M. Stone, J. Roy. Stat. Soc. B Met. 36, 111 (1974).

[28] D. Allen, Technometrics 16, 125 (1974).

[29] C. Wolverton, Acta Mater. 49, 3129 (2001).

[30] G. Kresse and J. Hafner, Phys. Rev. B 47, RC558 (1993).

[31] G. Kresse and J. Furthmüller, Comp. Mat. Sci. 6, 15 (1996).

[32] G. Kresse and J. Furthmüller, Phys. Rev. B 54, 11169 (1996).

[33] D. Vanderbilt, Phys. Rev. B 41, 7892 (1990).

[34] G. Kresse and J. Hafner, J. Phys. C: Condens. Matter 6, 8245 (1994).

[35] We used the Vienna ab initio simulation package 30, 31, 32] with ultra-soft pseudo-potentials 33] from Kresse and Hafner [34], a plane-wave cutoff of $440 \mathrm{eV}$, and a fine $k$ space mesh to ensure forces $<30 \mathrm{meV} / \AA$ and relative energy accuracy $\sim 1 \mathrm{meV} /$ atom. 